\title{
The effect of the area proportion of the metastatic lesion within the central metastatic lymph node on response to therapy in papillary thyroid carcinoma
}

\author{
LIUHONG SHI $^{1}$, LIANG ZHOU ${ }^{1}$, JIANBIAO WANG ${ }^{1}$, LEI JIN $^{1}$, YINJIAO LEI ${ }^{2}$, LIAN XIA $^{3}$ and LEI XIE ${ }^{1}$ \\ Departments of ${ }^{1}$ Head and Neck Surgery, ${ }^{2}$ Pathology and ${ }^{3}$ Operating Room Nursing, Affiliated Sir Run Run Shaw Hospital, \\ Zhejiang University School of Medicine, Zhejiang, Hangzhou 310016, P.R. China
}

Received June 27, 2020; Accepted December 18, 2020

DOI: $10.3892 / 01.2021 .12545$

\begin{abstract}
Lymph node (LN) metastasis has been strongly associated with locoregional recurrence and decreased survival time of patients with papillary thyroid carcinoma (PTC). Although the characteristics of the metastatic LNs (mLN) have been determined, including size, number, micro-metastasis and extra-nodal extension (ENE), further analysis is warranted. The present study introduced a new parameter known as the area proportion of the metastatic lesion within the central mLNs (APmCLN). The objective was to evaluate the impact of the APmCLN on response to therapy in patients with PTC. In total, 355 patients with PTC treated with total thyroidectomy and neck dissection, post-operative radioactive iodine and thyroid-stimulating hormone suppression were retrospectively studied. The patients were classified into two groups: Group A (APmCLN $\leq 75 \%$ ) and group B (APmCLN $>75 \%$ ). The association of various clinicopathological characteristics between these two groups was investigated. Univariate and multivariate analyses were used to evaluate risk factors associated with a non-Excellent response to therapy and recurrence-free survival (RFS). The analysis showed that APmCLN $>75 \%$ was significantly associated with extra-thyroidal extension, clinically apparent nodes (cN1), pathological N1b (pN1b), ENE, greater number and larger size of central mLN and larger size of the central LN metastatic lesion. Furthermore, it was reported that chronic lymphocytic thyroiditis, larger central mLN size and APmCLN >75\% were independent risk factors for a non-excellent response to therapy. Finally, it was determined that the rate of excellent response to therapy was significantly higher in pathological N1 (pN1) patients with
\end{abstract}

Correspondence to: Professor Lei Xie, Department of Head and Neck Surgery, Affiliated Sir Run Run Shaw Hospital, Zhejiang University School of Medicine, 3 Qing Chun Road East, Zhejiang, Hangzhou 310016, P.R. China

E-mail: xielsrrsh@zju.edu.cn

Key words: papillary thyroid carcinoma, area proportion, metastatic lesion, lymph node, response to therapy
APmCLN $\leq 75 \%(108 / 144,75.0 \%)$ compared with patients with APmCLN $>75 \%(27 / 47,57.4 \%)(\mathrm{P}=0.022)$. However, there was no significant difference $(\mathrm{P}=0.247)$ between patients with APmCLN $\leq 75 \%$ and pN0 $(132 / 164,80.5 \%)$. RFS was $89.4 \%$ in patients with pN1-APmCLN $>75 \%$, whereas those with pN1-APmCLN $\leq 75 \%$ and pN0 did not experience a relapse. Patients with PTC with APmCLN $>75 \%$ should be regarded as high-risk and may require more aggressive treatment and careful follow-up.

\section{Introduction}

Papillary thyroid carcinoma (PTC) is one of the most common endocrine malignancies and has a 10-year survival rate of $90-98 \%$ globally (1-4). Risk factors including age ( $>45$ years), male sex, larger tumor size and distant metastasis contribute to poor survival based on the Surveillance, Epidemiology and End Results (SEER) database (5). Despite the fact that the number of metastatic lymph nodes (mLNs) has been recognized as a negative prognostic factor, other features of mLNs have yet to be fully understood. Recently, the characteristics of mLNs, in particular, size, extra-nodal extension (ENE) and micro-metastasis $(<0.2 \mathrm{~cm}$ as the largest dimension of the metastatic lesion) were initially proposed by the 2015 updated version of the American Thyroid Association (ATA) guidelines (6-8).

PTC often metastasizes to lymph nodes (LNs), especially in the central neck region, and the size of the metastatic lesion within the LN tends to reflect the degree of disease progression $(9,10)$. However, the status of the metastatic lesion within a $\mathrm{mLN}$ varies to a large extent. This may include varying metastatic deposit sizes in different LNs of varying size $(8,11)$. Therefore, it is reasonable to be concerned with not only the metastatic lesion size, but also the location within the LN. To better reflect the disease progression of patients with PTC, the present study proposed a novel parameter: The area proportion of the metastatic lesion within the central mLN (APmCLN).

The post-operative risk of recurrence during follow-up should be estimated dynamically according to the response to therapy re-staging system (2015 version of the ATA guidelines) (7). Previous studies have suggested that the therapeutic response system is closely correlated with prognosis (12-16). 
The objective of the current study was to evaluate the impact of APmCLN on the response to therapy in patients with PTC.

\section{Materials and methods}

Patients. Between January 2013 and December 2015, 562 patients with PTC were enrolled onto the study at the Affiliated Sir Run Run Shaw Hospital (Zhejiang, China), and 355 of those underwent a total thyroidectomy (TT) with ipsilateral or bilateral central neck dissection (CND) (Fig. 1). Inclusion criteria were patients with pathologically diagnosed PTC. Exclusion criteria included no PTC diagnosis, poorly differentiated PTC (diagnostic criteria were based on the consensus Turin proposal) (6), coexisting other malignancies, previous thyroidectomy and no radioactive iodine (RAI) ablation. After surgery, a histological diagnosis of PTC and measurements of mLN were confirmed by two experienced pathologists from the Department of Pathology, who were independent from the present study. Of the 355 patients that met the final selection criteria, 191 and 164 were designated as pN1 and pN0, respectively (Fig. 1) (17). In the conventional Tumor-Node-Metastasis (TNM) staging system established by the Union for International Cancer Control and the American Joint Commission on Cancer (AJCC; 2010, 7th edition), pathologically confirmed lymph node metastasis was defined as pN1, no lymph node metastasis was defined as pNO. The study was approved by The Ethics Committee of the Affiliated Sir Run Run Shaw Hospital, Zhejiang University School of Medicine, and all enrolled patients provided written informed consent.

Treatment protocol. In accordance with the 2009 ATA guidelines (6), TT was performed if the patient met one of the following criteria: Bilateral nodularity, extra-thyroidal extension(ETE), tumor diameter $>1 \mathrm{~cm}$, mulifocal lesions in the affected lobe, regional or distant metastases, a personal history of radiation therapy to the head and neck or a first-degree family history of PTC. Ipsilateral CND was performed routinely for the affected side, regardless of whether the central neck LNs were clinically metastatic. Bilateral CND was performed for patients whose tumor(s) was located in the isthmus or both lobes, or for those with clinical metastasis in the neck LNs. Modified lateral neck dissection (LND), including levels II-IV or together with V, was performed only in patients with clinically evident nodal disease in the lateral neck on preoperative ultrasonography or when the ultrasound-guided fine needle aspiration of a lateral node exhibited positive results. RAI remnant ablation was performed postoperatively in all patients according to the 2009 ATA guidelines (6). Only the LNs from the central area were examined, which were derived from patients who underwent CND with or without LND.

Histopathological examination. Tissues with thyroid, tumor(s) and LN(s) were collected from each patient during surgery and were sent immediately to the Department of Pathology. Tissues were fixed in $10 \%$ neutral-buffered formalin at $4^{\circ} \mathrm{C}$ overnight, dehydrated using graded ethanol (100, 95, 75 and 50\%) and embedded in paraffin. If the thickness of the LNs was $<6$ $\mathrm{mm}$, they were embedded entirely in paraffin, cut in half, and then sliced into three 3-4- $\mu \mathrm{m}$ pieces. If a $\mathrm{LN}$ was $>6 \mathrm{~mm}$ in thickness, it was selected to be embedded and cut in half. All of the slices were stained with hematoxylin and eosin (H\&E) prior to pathological diagnosis (18). In the event of suspicious micro-metastasis, thyroglobulin ( $\mathrm{Tg}$ ) immunohistochemical (IHC) staining was performed $(19,20)$. For IHC, slides were incubated with a primary monoclonal rabbit anti-Thyroglobulin-antibody (1:300 dilution; Abcam; ab156008) at room temperature for $60 \mathrm{~min}$. Then, the slides were incubated with a secondary anti-rabbit IgG antibody (ImmPress Reagent Kit; MP-7405; peroxidase-conjugated) followed by target detection using DAB plus chromogen for $10 \mathrm{~min}$ (Gene Tex; GTX73338). All mLNs in the central neck were observed using light microscopy (magnification, x200-400) and measured by two experienced pathologists using an ocular micrometer, and the mean value of the measurements was recorded. In total, 2,768 central LNs were examined, of which 670 were positive, and the one with the largest size, lesion, or metastatic area was selected as the representative parameter for each patient.

Definitions. A new parameter (APmCLN) was identified and defined as the ratio of the metastatic deposit area to the whole $\mathrm{mLN}$ area in a cross-section, which was microscopically measured by a cross and divided into four quadrants: i) $\leq 25 \%$ (1 Quadrant occupied by the metastatic lesion), ii) $25-50 \%$ ( 2 quadrants occupied by the metastatic lesion), iii) $50-75 \%$ ( 3 quadrants occupied by the metastatic lesion) and iv) $>75 \%$ (4 quadrants almost occupied by the metastatic lesion) (Fig. 2). When evaluating independent risk factors for treatment response, there was no statistical significance between group i) and ii)+iii)+iv), groups i)+ii) and iii)+iv). However, differences between groups i) +ii) +iii) and iv) were statistically significant. Therefore, groups i)-iii) were merged into group A and group iv) was merged into group B.

Micro-metastasis was defined as the presence of metastatic deposits within a $\mathrm{LN}<2 \mathrm{~mm}$ in diameter, which is a parameter commonly used in breast cancer and other solid tumors (11). Micro-metastasis has been a proposed modification expressed in the 2015 ATA guidelines as a low-risk parameter (7). Patients presenting with histopathological criteria including diffuse infiltration of the thyroid gland with lymphocytes and other inflammation-related cells were diagnosed with chronic lymphocyte thyroiditis (CLT) (21).

The current definition of 'clinically apparent' LN metastasis (clinical N1 disease; cN1) includes any metastatic LN identified by palpation or imaging either before initial surgery or intraoperatively (8). When suspicious LN appeared at level VI or II-IV, it was defined as cN1a or $\mathrm{cN} 1 \mathrm{~b}$, respectively.

Assessment of treatment response. The response to the therapy re-staging system has been designed for the follow-up of patients with PTC in the 2015 ATA guidelines. According to biochemical, imaging and cyto-pathological findings, there are four response-to-therapy categories for patients treated with TT and RAI remnant ablation (7). These include: i) Excellent response, ii) biochemical incomplete response, iii) structural incomplete response and iv) indeterminate response. The current study rearranged these into two categories consisting of excellent response and non-excellent response. The latter included biochemical incomplete, structural incomplete and indeterminate responses. According to the ATA guidelines, the recurrence rate for the excellent response group is $1-4 \%$, which is much lower compared with other groups (biochemical 

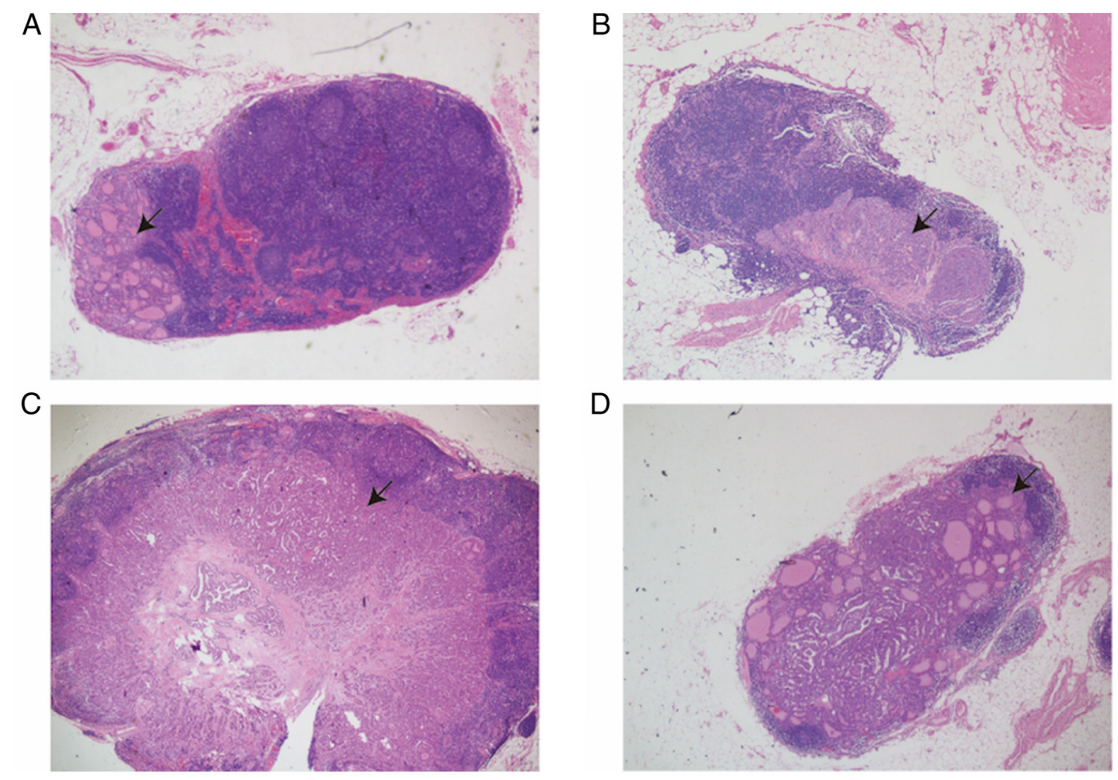

Figure 1. Representative hematoxylin/eosin-stained sections of central metastatic lymph nodes based on the area proportion of these lesions. (A) APmCLN $\leq 25 \%$ (20x magnification). (B) APmCLN $>25 \%$ and $\leq 50 \%$ (40x magnification). (C) APmCLN $>50 \%$ and $\leq 75 \%$ (20x magnification). (D) APmCLN $>75 \%$ (20x magnification). Black arrows indicate the metastatic lesions. APmCLN, area proportion of the metastatic lesion within the central metastatic lymph node.

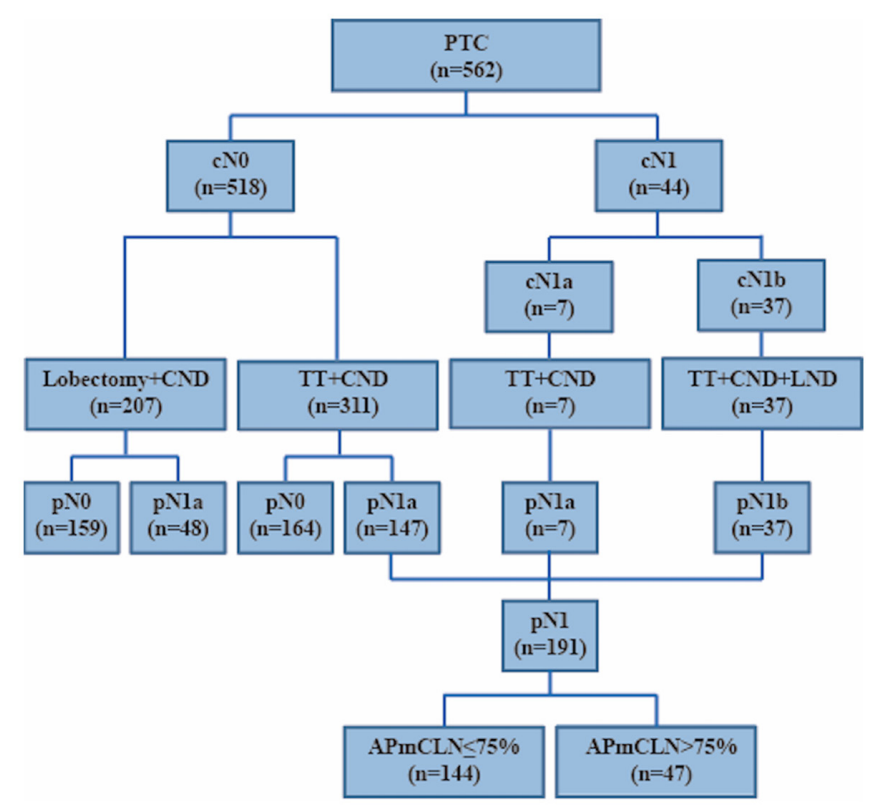

Figure 2. Flow diagram showing the included papillary thyroid carcinoma patients. PTC, papillary thyroid carcinoma; TT, total thyroidectomy; CND, central neck dissection; LND, lateral neck dissection; APmCLN, area proportion of the metastatic lesion within the central metastatic lymph node; c, clinically apparent; N, node; p, pathological.

incomplete response, $20 \%$ develop structural disease; structural incomplete response, $50-85 \%$ continue to have persistent disease despite additional therapy and indeterminate response, 15-20\% will have structural disease) (7).

All patients with PTC were followed up after completion of RAI remnant ablation. Routine neck ultrasound examination, and measurement of serum $\mathrm{Tg}$ and anti-thyroglobulin antibody ( $\mathrm{TgAb}$ ) were performed in a state of TSH (Thyroid Stimulating Hormone) suppression every 3 months in the first year and every 6-12 months thereafter. When biochemical or structural incomplete response occurred, more frequent follow-up was recommended with additional examinations (including CT scan and Fine Needle Aspiration) were proposed. The follow-up time for all PTCs ranged from 40 to 75 months (median, 57 months), and the assessment of response to therapy was based on the latest examination results. Serum $\mathrm{Tg}$ (normal range, 1.15-35.00 $\mathrm{ng} / \mathrm{ml}$ ) and $\mathrm{TgAb}$ (normal range, 0-4.11 IU/ml) were measured by electrochemiluminescence immunoassay in an Abbott Aeroset ${ }^{\circledR}$ Automated Instrument Analyzer (Canon Medical Systems Corp.) (22-24).

Statistical analysis. Continuous variables and categorical variables were determined by Mann-Whitney $U$ test and $\chi^{2}$ test (including Fisher's exact tests, if needed), respectively. Bonferroni's correction was also applied where appropriate. The results are presented as medians with range and numbers with percentages. Univariate logistic regression analyses were performed for sex, age, tumor size, tumor multifocality, ETE, CLT, $\mathrm{N}$ stage, number and size of central metastatic LN, the size of central metastatic lesion and APmCLN. The variables exhibiting $\mathrm{P}<0.05$ in the univariate analysis were then selected and analyzed using multivariate logistic regression analysis. The results were represented as odds ratios (ORs) with 95\% confidence intervals (CIs). For all analyses, two-sided tests were employed and $\mathrm{P}<0.05$ was considered to indicate a statistically significant difference. Statistical analyses were performed using SPSS software version 23.0 (IBM Corp.).

\section{Results}

Characteristics of patients with PTC with TT. The profiles of 562 patients are shown in Fig. 1. Among them, 207 underwent lobectomy, 355 underwent total thyroidectomy (TT), and these 355 patients included 311 cases of $\mathrm{cN0}$ and $44 \mathrm{cN} 1$. The clinicopathological characteristics of the 355 PTC patients are in Table I. The median age of the cohort was 42 years (range, 
13-72 years). Most of the patients were female (77.2\%) and were $<55$ years-old $(83.9 \%)$. The majority of patients $(234 / 355$, $65.9 \%$ ) were diagnosed with papillary thyroid micro-carcinoma (PTMC, primary tumor size $\leq 1 \mathrm{~cm}$ ). Multifocality, CLT, and ETE were found in 56.1 (199/355), 26.5 (94/355) and $32.4 \%(115 / 355)$ of patients, respectively. The clinical N stage included $311 \mathrm{cN0}$ (87.6\%), $7 \mathrm{cN1a}(2.0 \%)$ and $37 \mathrm{cN1b}(10.4 \%)$ patients, whereas the pathological $\mathrm{N}$ stage included $164 \mathrm{pN} 0$ (46.2\%), 154 pN1a (43.4\%) and and 37 pN1b (10.4\%) patients. An excellent response to primary therapy was observed in 267 patients $(75.2 \%)$. For ATA risk stratification, there were 157 (44.2\%), $100(28.2 \%)$ and $98(27.6 \%)$ patients in low-, intermediate- and high-risk categories, respectively.

Clinicopathological features associated with the APmCLN of patients with pN1-PTC. Comparison of the postoperative pathological results revealed several factors that were significantly different between group A (APmCLN $575 \%$ ) and group B (APmCLN $>75 \%)$. A larger APmCLN ( $75 \%)$ was associated with aggressive characteristics, including ETE $(\mathrm{P}=0.019)$, clinical node stage $(\mathrm{P}<0.001)$ and pathological node stage $(\mathrm{P}<0.001)$. To describe the features of the mLNs, a statistical analysis was performed for the number and size of the central mLN and the size and ENE of the metastatic foci. It was demonstrated that these factors were significantly different between the two groups ( $\mathrm{P}=0.001$ and $\mathrm{P}<0.001$, respectively). According to the 2015 ATA guidelines, the $\mathrm{mLN} \leq 5$ and micro-metastasis factors belong to the low-risk category. It was reported that these two factors were significantly higher in group A compared with group B (88.2 vs. $63.8 \%$ and 71.5 vs. $10.6 \%$, respectively; both $\mathrm{P}<0.001$ ). Therefore, in group $\mathrm{A}$, the proportion of low- and intermediate-risk categories was $70.1 \%$, whereas in group B, the proportion of intermediateand high-risk categories was $89.4 \%$. An APmCLN was associated with the risk stratification of disease recurrence (Table II).

Risk factors for non-excellent response to therapy of patients with pN1-PTC. Response to initial therapy was analyzed in all patients. The proportion of excellent responders in groups A and B were 75 and $57.4 \%$, respectively. There was a significant difference between the two groups $(\mathrm{P}=0.022$; Table II). The risk factors for non-excellent response to therapy in patients with pN1 PTC were further analyzed (Table III). Univariate analysis indicated that CLT $(\mathrm{P}<0.001)$, a higher quantity of central mLN $(\mathrm{P}=0.004)$, larger sizes of central $\mathrm{mLN}(\mathrm{P}<0.001)$ and their metastatic foci $(\mathrm{P}=0.025)$ and higher APmCLN $(\mathrm{P}=0.023)$ significantly increased the risk of classification into the non-excellent response to therapy category. Furthermore, CLT, size of central mLN and APmCLN were independent variables for response to therapy in multivariate analysis $(\mathrm{P}<0.00, \mathrm{P}=0.014$ and $\mathrm{P}=0.020$, respectively). Compared with cases without CLT, those with CLT were 5.405 times (95\% CI, 2.339-12.492; $\mathrm{P}<0.001)$ more likely to exhibit a non-excellent response to therapy. For cases with an incremental increase of $1 \mathrm{~mm}$ in central mLN size, the non-excellent risk increased by 1.283 times (95\% CI, 2.339-12.492; $\mathrm{P}=0.014$ ). For PTCs with APmCLN $>75 \%$, the rate was 3.917 times higher compared with that of APmCLN $\leq 75 \%$ (95\% CI, 1.245-12.327; P=0.020). Therefore, it was demonstrated that APmCLN ( $\leq 75$ vs. $>75 \%$ ) represents a new independent risk factor for predicting clinical outcome.
Table I. Characteristics of 355 patients with papillary thyroid carcinoma who underwent total thyroidectomy.

\begin{tabular}{|c|c|}
\hline Characteristics & Total \\
\hline \multicolumn{2}{|l|}{$\operatorname{Sex}^{\mathrm{a}}$} \\
\hline Male & $81(22.8)$ \\
\hline Female & $274(77.2)$ \\
\hline \multicolumn{2}{|l|}{ Age of diagnosis, years } \\
\hline Median (range), year & $42(13-72)$ \\
\hline$<55^{\mathrm{a}}$ & 298 (83.9) \\
\hline$\geq 55^{\mathrm{a}}$ & $57(16.1)$ \\
\hline \multicolumn{2}{|l|}{ Primary tumor size, $\mathrm{cm}^{\mathrm{a}}$} \\
\hline$\leq 1$ & $234(65.9)$ \\
\hline$>1$ & $121(34.1)$ \\
\hline \multicolumn{2}{|l|}{ Multifocality ${ }^{\mathrm{a}}$} \\
\hline Absent & $156(43.9)$ \\
\hline Present & $199(56.1)$ \\
\hline \multicolumn{2}{|l|}{$\mathrm{CLT}^{\mathrm{a}}$} \\
\hline Absent & $261(73.5)$ \\
\hline Present & $94(26.5)$ \\
\hline \multicolumn{2}{|l|}{ ETE $^{\mathrm{a}}$} \\
\hline Absent & $240(67.6)$ \\
\hline Present & $115(32.4)$ \\
\hline \multicolumn{2}{|l|}{ Clinical Node stage $^{a}$} \\
\hline $\mathrm{cNO}$ & $311(87.6)$ \\
\hline $\mathrm{cN} 1 \mathrm{a}$ & $7(2.0)$ \\
\hline $\mathrm{cN} 1 \mathrm{~b}$ & $37(10.4)$ \\
\hline \multicolumn{2}{|l|}{ Pathological Node stage ${ }^{\mathrm{a}}$} \\
\hline pNO & $164(46.2)$ \\
\hline pN1a & $154(43.4)$ \\
\hline $\mathrm{pN} 1 \mathrm{~b}$ & $37(10.4)$ \\
\hline \multicolumn{2}{|l|}{ Distant metastasis ${ }^{\mathrm{a}}$} \\
\hline Absent & $352(99.2)$ \\
\hline Present & $3(0.8)$ \\
\hline \multicolumn{2}{|l|}{ ATA response-to-therapy category ${ }^{a}$} \\
\hline Excellent response & $267(72.5)$ \\
\hline Biochemical incomplete response & $1(0.3)$ \\
\hline Structural incomplete response & $15(4.2)$ \\
\hline Indeterminate response & $72(20.3)$ \\
\hline \multicolumn{2}{|l|}{ ATA risk stratification $^{\mathrm{a}}$} \\
\hline Low & $157(44.2)$ \\
\hline Intermediate & $100(28.2)$ \\
\hline High & $98(27.6)$ \\
\hline
\end{tabular}

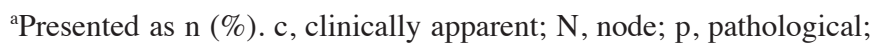
ATA, American Thyroid Association; ETE, extra-thyroidal extension; CLT, chronic lymphocyte thyroiditis.

Effect of the pathological metastasis of central LN on the Response to therapy category. It was observed that APmCLN affects the response to initial therapy in patients with pN1-PTC. The response to therapy in patients classified as pNO was further analyzed. A statistically significant difference in excellent 
Table II. Characteristics of 191 patients with pN1-papillary thyroid carcinoma according to the APmCLN.

\begin{tabular}{|c|c|c|c|}
\hline \multirow[b]{2}{*}{ Characteristics } & \multicolumn{2}{|c|}{ APmCLN } & \multirow[b]{2}{*}{ P-value } \\
\hline & Group A $\leq 75 \%, n=144$ & Group B $>75 \%, n=47$ & \\
\hline \multicolumn{4}{|l|}{$\operatorname{Sex}^{\mathrm{a}}$} \\
\hline Male & $37(25.7)$ & $19(40.4)$ & \multirow[t]{2}{*}{0.054} \\
\hline Female & $107(74.3)$ & $28(59.6)$ & \\
\hline \multicolumn{4}{|l|}{ Age of diagnosis, years } \\
\hline Median (range) & $39.5(20.0-69.0)$ & $37.0(25.0-66.0)$ & 0.786 \\
\hline$<55^{\mathrm{a}}$ & $126(87.5)$ & $44(93.6)$ & \multirow[t]{2}{*}{0.244} \\
\hline$\geq 55^{\mathrm{a}}$ & $18(12.5)$ & $3(6.4)$ & \\
\hline \multicolumn{4}{|l|}{ Primary tumor size, $\mathrm{cm}^{\mathrm{a}}$} \\
\hline$\leq 1$ & $84(58.3)$ & $22(46.8)$ & \multirow[t]{2}{*}{0.167} \\
\hline$>1$ & $60(41.7)$ & $25(53.2)$ & \\
\hline \multicolumn{4}{|l|}{ Multifocality ${ }^{\mathrm{a}}$} \\
\hline Absent & $62(43.1)$ & $21(44.7)$ & \multirow[t]{2}{*}{0.845} \\
\hline Present & $82(56.9)$ & $26(55.3)$ & \\
\hline \multicolumn{4}{|l|}{$\mathrm{ETE}^{\mathrm{a}}$} \\
\hline Absent & $95(66.0)$ & $22(46.8)$ & \multirow[t]{2}{*}{0.019} \\
\hline Present & $49(34.0)$ & $25(53.2)$ & \\
\hline \multicolumn{4}{|l|}{ CLT $^{\mathrm{a}}$} \\
\hline Absent & $108(75.0)$ & $39(83.0)$ & \multirow[t]{2}{*}{0.259} \\
\hline Present & $36(25.0)$ & $8(17.0)$ & \\
\hline \multicolumn{4}{|l|}{ Clinical Node stage ${ }^{\mathrm{a}}$} \\
\hline $\mathrm{cNO}$ & $123(85.4)$ & $24(51.1)$ & \multirow[t]{2}{*}{$<0.001$} \\
\hline $\mathrm{cN} 1$ & $21(14.6)$ & $23(48.9)$ & \\
\hline \multicolumn{4}{|l|}{ Pathological Node stage ${ }^{a}$} \\
\hline $\mathrm{pN} 1 \mathrm{a}$ & $128(88.9)$ & $26(55.3)$ & \multirow[t]{2}{*}{$<0.001$} \\
\hline $\mathrm{pN} 1 \mathrm{~b}$ & $16(11.1)$ & $21(44.7)$ & \\
\hline \multicolumn{4}{|l|}{ Number of central mLN } \\
\hline Median (range) & $2.0(1.0-19.0)$ & $5.0(1.0-18.0)$ & $<0.001$ \\
\hline$\leq 5^{\mathrm{a}}$ & $127(88.2)$ & $30(63.8)$ & \multirow[t]{2}{*}{$<0.001$} \\
\hline$>5^{\mathrm{a}}$ & 17(11.8) & $17(36.2)$ & \\
\hline \multicolumn{4}{|l|}{ Size of central mLN, mm } \\
\hline Median (range) & $4.33(0.67-14.90)$ & $6.40(1.07-18.27)$ & 0.001 \\
\hline \multicolumn{4}{|c|}{ Size of central LN metastatic foci, $\mathrm{mm}$} \\
\hline Median (range) & $1.34(0.07-6.80)$ & $5.33(1.07-18.27)$ & $<0.001$ \\
\hline$<2^{\mathrm{a}}$ & $103(71.5)$ & $5(10.6)$ & \multirow[t]{2}{*}{$<0.001$} \\
\hline$\geq 2^{\mathrm{a}}$ & $41(28.5)$ & $42(89.4)$ & \\
\hline $\mathrm{ENE}^{\mathrm{a}}$ & & & \\
\hline Absent & $135(93.8)$ & $30(63.8)$ & $<0.001$ \\
\hline Present & $9(6.2)$ & $17(36.2)$ & \\
\hline ATA response-to-therapy & & & \\
\hline Excellent response & $108(75.0)$ & $27(57.4)$ & 0.022 \\
\hline Non-excellent response & $36(25.0)$ & $20(42.6)$ & \\
\hline ATA risk stratification $^{\mathrm{a}}$ & & & \\
\hline Low & $52(36.1)$ & $5(10.6)$ & 0.003 \\
\hline Intermediate & $49(34.0)$ & $19(40.4)$ & \\
\hline High & 43 (29.9) & $23(49.0)$ & \\
\hline
\end{tabular}

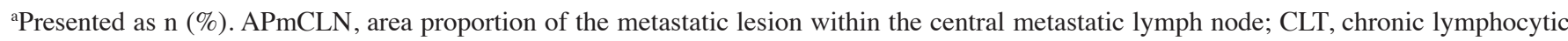
thyroiditis; ETE, extra-thyroidal extension; LN, lymph node; mLN, metastatic lymph node; ENE, extra-nodal extension; ATA, American Thyroid Association; c, clinically apparent; N, node; p, pathological. 
Table III. Relationships between clinicopathological variables and non-excellent response-to-therapy in patients with pN1-papillary thyroid carcinoma.

\begin{tabular}{llrr}
\hline & \multicolumn{1}{c}{ Univariate } & & Multivariate \\
\cline { 2 - 3 } Characteristics & OR $(95 \%$ CI $)$ & P-value & OR (95\% CI) \\
\hline Sex, male vs. female & $1.546(0.754-3.171)$ & 0.235 & \\
Age of diagnosis, $<55$ vs. $\geq 55$ years & $0.534(0.171-1.664)$ & 0.279 & \\
Primary tumor size, $\leq 1$ vs. $>1$ cm & $1.116(0.597-2.087)$ & 0.730 & \\
Multifocality, absent vs. present & $1.755(0.918-3.356)$ & 0.089 & \\
ETE, absent vs. present & $0.833(0.437-1.590)$ & 0.580 & \\
CLT, absent vs. present & $6.462(3.114-13.413)$ & $<0.001$ & $5.405(2.339-12.492)$ \\
Pathological N stage, pN1a vs. pN1b & $1.623(0.764-3.447)$ & 0.207 & \\
Clinical N stage, cN0 vs. cN1 & $1.529(0.749-3.120)$ & 0.244 & \\
Number of central mLN, $\leq 5$ vs. $>5$ & $3.026(1.410-6.493)$ & 0.004 & $2.082(0.821-5.280)$ \\
Size of central mLN, mm & $1.216(1.103-1.341)$ & $<0.001$ & $1.283(1.051-1.566)$ \\
Size of central LN metastatic foci, mm & $1.110(1.013-1.215)$ & 0.025 & $0.823(0.652-1.039)$ \\
APmCLN, $\leq 25$ vs. $>25 \%$ & $1.148(0.610-2.158)$ & 0.669 & \\
APmCLN, $\leq 50$ vs. $>50 \%$ & $1.826(0.973-3.428)$ & 0.061 & \\
APmCLN, $\leq 75$ vs. $>75 \%$ & $2.222(1.114-4.432)$ & 0.023 & $3.917(1.245-12.327)$ \\
ENE, absent vs. present & $1.083(0.441-2.659)$ & 0.861 & 0.123 \\
\hline APmCLN, & & 0.102 \\
\end{tabular}

APmCLN, area proportion of the metastatic lesion within the central metastatic lymph node; CLT, chronic lymphocytic thyroiditis; ETE, extra-thyroidal extension; LN, lymph node; $\mathrm{mLN}$, metastatic lymph node; c, clinically apparent; $\mathrm{N}$, node; $\mathrm{p}$, pathological.

Table IV. Response to therapy categories on the pathological metastasis of central lymph node of patients with papillary thyroid carcinoma treated with total thyroidectomy.

\begin{tabular}{|c|c|c|c|}
\hline \multirow[b]{2}{*}{ Characteristics } & \multicolumn{2}{|c|}{ ATA response-to-therapy categories, n (\%) } & \multirow[b]{2}{*}{ P-value } \\
\hline & Excellent & Non-Excellent & \\
\hline pNO & $132(80.5)$ & $32(19.5)$ & $0.247^{\mathrm{a}}$ \\
\hline pN1-APmCLN $\leq 75 \%$ & $108(75.0)$ & $36(25.0)$ & $0.022^{\mathrm{b}}$ \\
\hline pN1-APmCLN >75\% & $27(57.4)$ & $20(42.6)$ & \\
\hline
\end{tabular}

${ }^{\mathrm{a}} \chi^{2}$ test for $\mathrm{pN} 0$ vs. APmCLN $<75 \%$ of $\mathrm{pN} 1$; ${ }^{\mathrm{b}} \chi^{2}$ test for APmCLN $\leq 75 \%$ vs. APmCLN $>75 \%$ of pN1. APmCLN, area proportion of the metastatic lesion within the central metastatic lymph node.

response to therapy was observed between patients in the APmCLN $\leq 75 \%$ and APmCLN $>75 \%(P=0.022)$ categories, after using Bonferroni's correction, it still passed the statistical significance $(\mathrm{P}=0.022<0.050 / 2)$. The excellent response to therapy rates were 75 and $57.4 \%$, respectively. Notably, when comparing the pN0 and pN1-APmCLN $\leq 75 \%$ groups, there was no statistical difference in the rate of achieving an excellent response to treatment $(\mathrm{P}=0.247)$, and the incidence rates for these two groups were 80.5 and $75.0 \%$, respectively (Table IV). Therefore, it is reasonable to believe that APmCLN $>75 \%$ will make Response to therapy worse than APmCLN $\leq 75 \%$.

Recurrence-free survival (RFS) according to the APmCLN. In total, five cases of disease recurrence were identified during the median follow-up period of 57 months (range, 40-75 months) across all patients with PTC who underwent
TT. The mean time of recurrence was 25.8 months. According to the APmCLN, the number of recurrence cases was higher in the pN1-APmCLN $>75 \%$ group compared with the pN1-APmCLN $\leq 75 \%$ group (5/47 vs. 0/144, respectively), and the RFS was also significantly different between the two groups (89.4 vs. $100 \%$; log-rank $\mathrm{P}<0.001)$. In addition, RFS of the pN1-APmCLN $\leq 75 \%$ and pN0 patients were both $100 \%$ and no patients relapsed (Fig. 3). Therefore, pN1-APmCLN $>75 \%$ indicates a higher recurrence rate in PTC patients.

\section{Discussion}

PTC is generally an indolent disease that has an excellent prognosis. The SEER registry study concluded that the overall survival rate at 14 years is $82 \%$ in cases of PTC without LN metastases and 79\% with nodal metastases in America (25). 


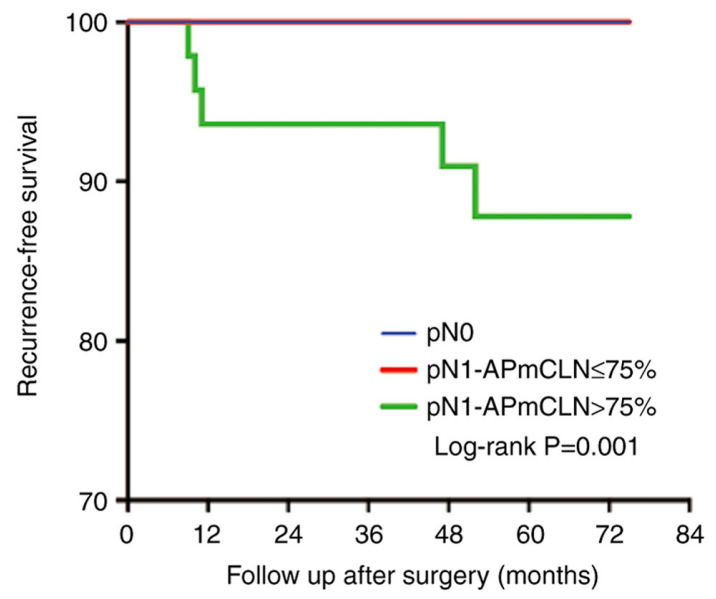

No. at risk

$\begin{array}{lllllllll}\text { pNO } & 164 & 164 & 164 & 164 & 164 & 164 & 164 & 164 \\ \text { pN1-APmCLN } \leq 75 \% & 144 & 144 & 144 & 144 & 144 & 144 & 144 & 144 \\ \text { pN1-APmCLN }>75 \% & 47 & 44 & 44 & 44 & 43 & 42 & 42 & 42\end{array}$

Figure 3. Kaplan-Meier curves showing recurrence-free survival according to the APmCLN. APmCLN, area proportion of the metastatic lesion within the central metastatic lymph node; $\mathrm{N}$, node; $\mathrm{p}$, pathological.

Therefore, an understanding of which patients require long-term follow-up for assessing disease status is needed. Methods to reduce the risk of recurrence and disease-specific death is also important. Previous studies have shown that the evaluation of clinical outcome in patients with PTC should be dynamically adjusted throughout the observation period (26-29). A key step in risk re-evaluation is to assess the response to primary therapy and analyze the clinical data obtained from imaging, biochemical and cytopathological examination during dynamic monitoring, especially within the first 2 years of follow-up $(7,15)$. According to the ATA guidelines, the response to therapy re-classification describes differences in clinical status and outcomes at various points during the observed progress period (7). For example, one study demonstrated a recurrence rate of $1-4 \%$ in the excellent response group, while for the remaining three groups, the likelihood of disease recurrence, persistence or progression is significantly increased (7).

The weight of LN metastasis has been decreased in the assessment of the new TNM system in patients with PTC. The nodal status does not affect TNM staging in patients $<55$ years old. In patients $>55$ years old with $\mathrm{T} 1$ or $\mathrm{T} 2$, $\mathrm{N} 1$ can promote the stage from I to II (17). However, LN metastasis in the central neck occurs frequently in PTC (10) and correlates with locoregional recurrence $(8,30)$. Therefore, for the assessment of recurrent risk according to the 2015 ATA guidelines, the extent of LN involvement, including the number and size of mLN and LN metastasis, was added and emphasized (7). In previous years, some researchers have been concerned with the impact of other mLN parameters on PTC biological behavior and prognosis, such as ENE (31), cancerous nodules of LNs (32) and the ratio of metastatic LNs to total LNs removed $(33,34)$. In the present study, APmCLN was initially introduced as a new parameter and its effect on PTC prognosis was analyzed.

Although five mLNs in cN0, micro-metastasis and the central mLN (mCLN) size were added to the 2015 ATA guidelines as new factors of recurrence risk assessment, the two parameters were not found to affect the response of initial treatment in the current study. The risk factors of non-excellent response to therapy were also evaluated using univariate and multivariate analyses. The presence of CLT, larger size mCLN and APmCLN >75\% were independent risk factors for non-excellent response to therapy in patients with PTC with pN1.

There is no definitive conclusion as to whether CLT is linked with a particular set of clinicopathological characteristics or prognosis in PTC. Some studies have shown that multifocality and LN metastasis occurs more frequently in patients with CLT (35-37). In contrast, other studies found associations between the presence of CLT and improved features, such as smaller tumor size, less advanced TNM stage, lower frequency of LN metastasis and improved prognosis (38-40). In the present study, CLT was recognized as a risk factor for non-excellent response to therapy in PTC. One of the characteristics of CLT is abnormally elevated $\mathrm{TgAb}$ that does not return to normal within 2 years in some patients after TT (41-43). The increased $\mathrm{TgAb}$ may interfere with the measurement of $\mathrm{Tg}$, which would affect evaluation of the initial treatment response (44-46). Therefore, it was hypothesized that CLT may not be suitable for predicting treatment response.

The size of the mCLN is an important prognostic factor for patients with PTC, and larger mCLNs tend to be more aggressive. Ito et al (47) showed that the presence of $m L N>1.5 \mathrm{~cm}$ is associated with a significantly lower disease-free survival rate compared with patients with either N0 disease or patients with pN1 disease $<1.5 \mathrm{~cm}$. Similarly, Sugitani et al (48) reported that in patients with $\mathrm{pN} 1$ disease with the largest $\mathrm{mLN}(>3 \mathrm{~cm}$, 27\%), the risk of recurrence within 10 years after TT and neck dissection without RAI ablation was significantly higher compared with $\mathrm{pN} 1$ patients $(<3 \mathrm{~cm}, 11 \%)$. In the present study, larger-sized central mLN was significantly associated with an increased risk of non-excellent response to therapy. However, because of the long-term stimulation of inflammation, the central LNs of CLT are relatively larger. Therefore, it may be unreasonable to consider the larger $\mathrm{MCLN}$ size as an independent risk factor for poor treatment response if the inference factor of CLT is not ruled out.

To better reflect the metastatic extent of LNs, the present study put forward the novel parameter of APmCLN, which includes two factors. These are the size of the metastatic LN and its metastatic foci, and whether the influence of inflammation due to CLT may be ruled out. This was conducted so that the definition of APmCLN is more scientific and rational. Previous studies have reported that the sizes of mLN and LN metastasis are indicators of tumor aggressiveness and risk factors for prognosis in PTC (47,49-51). However, coexisting CLT, the frequently changing size of mCLN changes and the size of $\mathrm{mLN}$ foci were not independent risk factors for non-excellent response to therapy in the current study. It is inaccurate to predict treatment response based only on CLT and the size of mLNs, therefore the present study included APmCLN and systematically analyzed its clinicopathological and prognostic value in patients with pN1-PTC after TT and CND. APmCLN $>75 \%$ exhibited worse clinicopathological features, a poor prognosis and may be regarded as 
a new independent risk factor for non-excellent response to therapy. Therefore, there was sufficient data to conclude that APmCLN may be a reliable and practical new indicator for predicting response to therapy and prognosis in patients with pN1-PTC.

Several limitations were evident in the current study. First, there were only 355 patients enrolled and only five relapsed cases were reported in the relatively short follow-up period. Therefore, additional studies with longer follow-up times and multicenter data are needed. Second, APmCLN is currently a categorical variable. The $75 \%$ critical value is obtained through step classification and comparison between groups. If it is a continuous variable, the cut-off value can be calculated using an ROC curve (52-54), which is more scientific. Given the differences in the color and cell morphology of H\&E-stained sections between tumor and normal tissue, software, such as Image J and ITK-SNAP (55-58), can be used to identify the boundary, obtain the location and range of metastases in the $\mathrm{LN}$, and calculate the area proportion of the metastatic lesion. This more extensive analysis would allow APmCLN to be analyzed as a continuous variable. Third, according to the ATA guidelines (2015 version) (7), some patients with PTMC are recommend Active Surveillance rather than surgery. Active Surveillance is applying life-long diagnostic modalities to evaluate changes in disease status without treatment, until progression of the disease becomes clinically apparent (59-61). Regular follow-up should be provided for the patient to ensure that disease progression is tolerable without any additional therapeutic options, such as surgery. However, due to the retrospective nature of the present study, most of the patients enrolled had undergone surgical treatment, which was inconsistent with the current ATA guidelines (7). The present patients were recruited between 2013 and 2015, and treated according to the 2009 ATA guidelines (6) and the Chinese Thyroid Association (62) in which thyroid surgery is recommended if the lesion is considered to be malignant.

In conclusion, APmCLN $>75 \%$ was an indicator of tumor aggressiveness and a significant independent risk factor for non-excellent response to therapy in patients with pN1-PTC. These results may enable physicians to further stratify patients into various risk groups and develop effective individual follow-up and treatment plans.

\section{Acknowledgements}

Not applicable.

\section{Funding}

This research was supported by The Innovative Talent Support Program of Medicine and Health Technology project from the Zhejiang Health Commission (grant no. 2021RC078) and The General Research Project of Zhejiang Provincial Department of Education (grant no. Y202043478).

\section{Availability of data and materials}

The datasets used and/or analyzed during the current study are available from the corresponding author on reasonable request.

\section{Authors' contributions}

LHS and LeX designed the study and confirm the authenticity and legitimacy of all raw data. LHS wrote the manuscript. LZ, JW and LJ performed the data collection and analysis. LHS interpreted the results. YL performed the histological examination, and confirmed the diagnosis. LiX performed the follow-up plan and collected the data from patients. LeX supervised the project. All authors read and approved the final manuscript.

\section{Ethics approval and consent to participate}

This study was approved by The Ethics Committee of the Affiliated Sir Run Run Shaw Hospital, Zhejiang University School of Medicine (Hangzhou, China). All patients provided written informed consent.

\section{Patient consent for publication}

Not applicable.

\section{Competing interests}

The authors declare that they have no competing interests.

\section{References}

1. Bilimoria KY, Bentrem DJ, Ko CY, Stewart AK, Winchester DP, Talamonti MS and Sturgeon C: Extent of surgery affects survival for papillary thyroid cancer. Ann Surg 246: 375-384, 2007.

2. Adam MA, Pura J, Gu L, Dinan MA, Tyler DS, Reed SD, Scheri R, Roman SA and Sosa JA: Extent of surgery for papillary thyroid cancer is not associated with survival: An analysis of 61,775 patients. Ann Surg 260: 601-607, 2014.

3. Barney BM, Hitchcock YJ, Sharma P, Shrieve DC and Tward JD: Overall and cause-specific survival for patients undergoing lobectomy, near-total, or total thyroidectomy for differentiated thyroid cancer. Head Neck 33: 645-649, 2011.

4. Gilliland FD, Hunt WC, Morris DM and Key CR: Prognostic factors for thyroid carcinoma. A population-based study of 15,698 cases from the Surveillance, Epidemiology and End Results (SEER) program 1973-1991. Cancer 79: 564-573, 1997.

5. Doll KM, Rademaker A and Sosa JA: Practical guide to surgical data sets: Surveillance, epidemiology, and end results (SEER) database. JAMA Surg 153: 588-589, 2018.

6. American Thyroid Association (ATA) Guidelines Taskforce on Thyroid Nodules and Differentiated Thyroid Cancer; Cooper DS, Doherty GM, Haugen BR, Kloos RT, Lee SL, Mandel SJ, Mazzaferri EL, McIver B, Pacini F, et al: Revised American Thyroid Association management guidelines for patients with thyroid nodules and differentiated thyroid cancer. Thyroid 19: 1167-1214, 2009.

7. Haugen BR, Alexander EK, Bible KC, Doherty GM, Mandel SJ, Nikiforov YE, Pacini F, Randolph GW, Sawka AM, Schlumberger M, et al: 2015 American Thyroid Association Management Guidelines for Adult Patients with Thyroid Nodules and Differentiated Thyroid Cancer: The American Thyroid Association Guidelines Task Force on Thyroid Nodules and Differentiated Thyroid Cancer. Thyroid 26: 1-133, 2016.

8. Randolph GW, Duh QY, Heller KS, LiVolsi VA, Mandel SJ, Steward DL, Tufano RP and Tuttle RM; American Thyroid Association Surgical Affairs Committee's Taskforce on Thyroid Cancer Nodal Surgery: The prognostic significance of nodal metastases from papillary thyroid carcinoma can be stratified based on the size and number of metastatic lymph nodes, as well as the presence of extranodal extension. Thyroid 22: 1144-1152,2012.

9. Roh JL, Park JY and Park CI: Total thyroidectomy plus neck dissection in differentiated papillary thyroid carcinoma patients: Pattern of nodal metastasis, morbidity, recurrence, and postoperative levels of serum parathyroid hormone. Ann Surg 245: 604-610, 2007. 
10. Sivanandan R and Soo KC: Pattern of cervical lymph node metastases from papillary carcinoma of the thyroid. Br J Surg 88: 1241-1244, 2001.

11. Cranshaw IM and Carnaille B: Micrometastases in thyroid cancer. An important finding? Surg Oncol 17: 253-258, 2008

12. Raue F and Frank-Raue K: Thyroid cancer: Risk-stratified management and individualized therapy. Clin Cancer Res 22: 5012-5021, 2016

13. Tuttle RM, Tala H, Shah J, Leboeuf R, Ghossein R, Gonen M, Brokhin M, Omry G, Fagin JA and Shaha A: Estimating risk of recurrence in differentiated thyroid cancer after total thyroidectomy and radioactive iodine remnant ablation: Using response to therapy variables to modify the initial risk estimates predicted by the new American Thyroid Association staging system. Thyroid 20: 1341-1349, 2010

14. Castagna MG, Maino F, Cipri C, Belardini V, Theodoropoulou A, Cevenini G and Pacini F: Delayed risk stratification, to include the response to initial treatment (surgery and radioiodine ablation), has better outcome predictivity in differentiated thyroid cancer patients. Eur J Endocrinol 165: 441-446, 2011.

15. Ruben R, Pavithran PV, Menon VU, Nair V and Kumar H: Performance of ATA risk stratification systems, response to therapy, and outcome in an indian cohort of differentiated thyroid carcinoma patients: A retrospective study. Eur Thyroid J 8: 312-318, 2019.

16. Tuttle RM and Alzahrani AS: Risk stratification in differentiated thyroid cancer: From detection to final follow-up. J Clin Endocrinol Metab 104: 4087-4100, 2019.

17. Edge SB and Compton CC: The American Joint Committee on Cancer: The 7th edition of the AJCC cancer staging manual and the future of TNM. Ann Surg Oncol 17: 1471-1474, 2010

18. Bassotti G, Villanacci V, Salerni B, Maurer CA and Cathomas G: Beyond hematoxylin and eosin: The importance of immunohistochemical techniques for evaluating surgically resected constipated patients. Tech Coloproctol 15: 371-375, 2011.

19. Kaczka K, Wojcik I, Matejkowska M, Kuzdak K and Pomorski L: Lymph node metastases of papillary thyroid cancer in immunohistochemical and molecular examination-preliminary report. Endokrynol Pol 56: 160-167, 2005 (In Polish).

20. Stavropoulos A, Varras M, Vasilakaki T, Varra VK, Tsavari A Varra FN, Nonni A, Kavantzas N and Lazaris AC: Expression of p53 and PTEN in human primary endometrial carcinomas: Clinicopathological and immunohistochemical analysis and study of their concomitant expression. Oncol Lett 17: 4575-4589, 2019.

21. Rho MH, Kim DW, Hong HP, Park YM, Kwon MJ, Jung SJ, Kim YW and Kang T: Diagnostic value of antithyroid peroxidase antibody for incidental autoimmune thyroiditis based on histopathologic results. Endocrine 42: 647-652, 2012.

22. Spencer CA: Clinical review: Clinical utility of thyroglobulin antibody $(\mathrm{Tg} \mathrm{Ab})$ measurements for patients with differentiated thyroid cancers (DTC). J Clin Endocrinol Metab 96: 3615-3627, 2011.

23. Segurado OG, Volmer W and Dowell B: PSA standardization: A review of NCCLS, Stanford and Abbott efforts. Anticancer Res 17: 2919-2920, 1997.

24. Feldt-Rasmussen U and Rasmussen AK: Serum thyroglobulin $(\mathrm{Tg})$ in presence of thyroglobulin autoantibodies $(\mathrm{Tg} \mathrm{Ab})$. Clinical and methodological relevance of the interaction between $\mathrm{Tg}$ and $\mathrm{Tg} \mathrm{Ab}$ in vitro and in vivo. J Endocrinol Invest 8: 571-576, 1985.

25. Podnos YD, Smith D, Wagman LD and Ellenhorn JD: The implication of lymph node metastasis on survival in patients with well-differentiated thyroid cancer. Am Surg 71: 731-734, 2005.

26. Brito JP, Al Nofal A, Montori VM, Hay ID and Morris JC: The impact of subclinical disease and mechanism of detection on the rise in thyroid cancer incidence: A population-based study in olmsted county, minnesota during 1935 through 2012. Thyroid 25: 999-1007, 2015.

27. Aschebrook-Kilfoy B, Schechter RB, Shih YC, Kaplan EL, Chiu BC, Angelos P and Grogan RH: The clinical and economic burden of a sustained increase in thyroid cancer incidence. Cancer Epidemiol Biomarkers Prev 22: 1252-1259, 2013.

28. Harrison MB, Graham ID, van den Hoek J, Dogherty EJ, Carley ME and Angus V: Guideline adaptation and implementation planning: A prospective observational study. Implement Sci 8: 49, 2013

29. Silberstein EB, Alavi A,Balon HR, Clarke SE,DivgiC, Gelfand MJ, Goldsmith SJ, Jadvar H, Marcus CS, Martin WH, et al: The SNMMI practice guideline for therapy of thyroid disease with 131I 3.0. J Nucl Med 53: 1633-1651, 2012.
30. Baek SK, Jung KY, Kang SM, Kwon SY, Woo JS, Cho SH and Chung EJ: Clinical risk factors associated with cervical lymph node recurrence in papillary thyroid carcinoma. Thyroid 20: $147-152,2010$

31. Ricarte-Filho J, Ganly I, Rivera M, Katabi N, Fu W, Shaha A, Tuttle RM, Fagin JA and Ghossein R: Papillary thyroid carcinomas with cervical lymph node metastases can be stratified into clinically relevant prognostic categories using oncogenic BRAF, the number of nodal metastases, and extra-nodal extension. Thyroid 22: 575-584, 2012.

32. Chen L, Zhu Y, Zheng K, Zhang H, Guo H, Zhang L, Wu K, Kong L, Ruan W, Hu J, et al: The presence of cancerous nodules in lymph nodes is a novel indicator of distant metastasis and poor survival in patients with papillary thyroid carcinoma. J Cancer Res Clin Oncol 143: 1035-1042, 2017.

33. Lee J, Lee SG, Kim K, Yim SH, Ryu H, Lee CR, Kang SW, Jeong JJ, Nam KH, Chung WY and Jo YS: Clinical value of lymph node ratio integration with the 8th edition of the UICC TNM classification and 2015 ATA risk stratification systems for recurrence prediction in papillary thyroid cancer. Sci Rep 9: $13361,2019$.

34. Ryu IS, Song CI, Choi SH, Roh JL, Nam SY and Kim SY: Lymph node ratio of the central compartment is a significant predictor for locoregional recurrence after prophylactic central neck dissection in patients with thyroid papillary carcinoma. Ann Surg Oncol 21: 277-283, 2014.

35. Boi F, Pani F, Calo PG, Lai ML and Mariotti S: High prevalence of papillary thyroid carcinoma in nodular Hashimoto's thyroiditis at the first diagnosis and during the follow-up. J Endocrinol Invest 41: 395-402, 2018.

36. Resende de Paiva C, Grønhøj C, Feldt-Rasmussen U and von Buchwald C: Association between Hashimoto's thyroiditis and thyroid cancer in 64,628 patients. Front Oncol 7: 53, 2017.

37. Dong S, Xie XJ, Xia Q and Wu YJ: Indicators of multifocality in papillary thyroid carcinoma concurrent with Hashimoto's thyroiditis. Am J Cancer Res 9: 1786-1795, 2019.

38. Borowczyk M, Janicki A, Dworacki G, Szczepanek-Parulska E, Danieluk M, Barnett J, Antonik M, Kałużna M, Bromińska B, Czepczyński R, et al: Decreased staging of differentiated thyroid cancer in patients with chronic lymphocytic thyroiditis. J Endocrinol Invest 42: 45-52, 2019.

39. Ieni A, Vita R, Magliolo E, Santarpia M, Di Bari F, Benvenga S and Tuccari G: One-third of an archivial series of papillary thyroid cancer (Years 2007-2015) Has coexistent chronic lymphocytic thyroiditis, which is associated with a more favorable tumor-node-metastasis staging. Front Endocrinol (Lausanne) 8: 337, 2017.

40. Lun Y, Wu X, Xia Q, Han Y, Zhang X, Liu Z, Wang F, Duan Z, Xin $\mathrm{S}$ and Zhang $\mathrm{J}$ : Hashimoto's thyroiditis as a risk factor of papillary thyroid cancer may improve cancer prognosis. Otolaryngol Head Neck Surg 148: 396-402, 2013.

41. Kim WG, Yoon JH, Kim WB, Kim TY, Kim EY, Kim JM, Ryu JS, Gong G, Hong SJ and Shong YK: Change of serum antithyroglobulin antibody levels is useful for prediction of clinical recurrence in thyroglobulin-negative patients with differentiated thyroid carcinoma. J Clin Endocrinol Metab 93: 4683-4689, 2008

42. Latrofa F, Ricci D, Montanelli L, Rocchi R, Piaggi P, Sisti E, Grasso L, Basolo F, Ugolini C, Pinchera A and Vitti P: Lymphocytic thyroiditis on histology correlates with serum thyroglobulin autoantibodies in patients with papillary thyroid carcinoma: Impact on detection of serum thyroglobulin. J Clin Endocrinol Metab 97: 2380-2387, 2012.

43. Spencer C and Fatemi S: Thyroglobulin antibody ( TgAb) methods-Strengths, pitfalls and clinical utility for monitoring $\mathrm{Tg}$ Ab-positive patients with differentiated thyroid cancer. Best Pract Res Clin Endocrinol Metab 27: 701-712, 2013.

44. Jankovic B, Le KT and Hershman JM: Clinical Review: Hashimoto's thyroiditis and papillary thyroid carcinoma: Is there a correlation? J Clin Endocrinol Metab 98: 474-482, 2013.

45. Fugazzola L, Colombo C, Perrino M and Muzza M: Papillary thyroid carcinoma and inflammation. Front Endocrinol (Lausanne) 2: 88, 2011.

46. Shi L, Zhou L, Wang J, Li F and Xie L: Cytokine production of papillary thyroid carcinoma coexisting with Hashimoto's thyroiditis. Int J Clin Exp Pathol 10: 9567-9574, 2017.

47. Ito Y, Higashiyama T, Takamura Y, Miya A, Kobayashi K, Matsuzuka F, Kuma K and Miyauchi A: Risk factors for recurrence to the lymph node in papillary thyroid carcinoma patients without preoperatively detectable lateral node metastasis: Validity of prophylactic modified radical neck dissection. World J Surg 31: 2085-2091, 2007. 
48. Sugitani I, Kasai N, Fujimoto Y and Yanagisawa A: A novel classification system for patients with PTC: Addition of the new variables of large ( $3 \mathrm{~cm}$ or greater) nodal metastases and reclassification during the follow-up period. Surgery 135: 139-148, 2004.

49. Perrier ND, Brierley JD and Tuttle RM: Differentiated and anaplastic thyroid carcinoma: Major changes in the American Joint Committee on Cancer eighth edition cancer staging manual. CA Cancer J Clin 68: 55-63, 2018.

50. Jeon MJ, Yoon JH, Han JM, Yim JH, Hong SJ, Song DE, Ryu JS, Kim TY, Shong YK and Kim WB: The prognostic value of the metastatic lymph node ratio and maximal metastatic tumor size in pathological N1a papillary thyroid carcinoma. Eur J Endocrinol 168: 219-225, 2013

51. Lee SR, Kim HO, Son BH, Shin JH and Yoo CH: Prognostic significance of the metastatic lymph node ratio in patients with gastric cancer. World J Surg 36: 1096-1101, 2012.

52. Yu J, Yang L, Vexler A and Hutson AD: Easy and accurate variance estimation of the nonparametric estimator of the partial area under the ROC curve and its application. Stat Med 35: 2251-2282, 2016.

53. Sorribas A, March J and Trujillano J: A new parametric method based on S-distributions for computing receiver operating characteristic curves for continuous diagnostic tests. Stat Med 21: 1213-1235, 2002.

54. Habibzadeh F, Habibzadeh P and Yadollahie M: On determining the most appropriate test cut-off value: The case of tests with continuous results. Biochem Med (Zagreb) 26: 297-307, 2016.

55. Wang LW, Qu AP, Yuan JP, Chen C, Sun SR, Hu MB, Liu J and Li Y: Computer-based image studies on tumor nests mathematical features of breast cancer and their clinical prognostic value. PLoS One 8: e82314, 2013.

56. Dello SA, van Dam RM, Slangen JJ, van de Poll MC, Bemelmans MH, Greve JW, Beets-Tan RG, Wigmore SJ and Dejong CH: Liver volumetry plug and play: Do it yourself with ImageJ. World J Surg 31: 2215-2221, 2007.
57. Zhou J, Zhang Y, Chang KT, Lee KE, Wang O, Li J, Lin Y, Pan Z, Chang P, Chow D, et al: Diagnosis of benign and malignant breast lesions on DCE-MRI by using radiomics and deep learning with consideration of peritumor tissue. J Magn Reson Imaging 51: 798-809, 2020.

58. Nie P, Yang G, Wang Z, Yan L, Miao W, Hao D, Wu J, Zhao Y, Gong A, Cui J, et al: A CT-based radiomics nomogram for differentiation of renal angiomyolipoma without visible fat from homogeneous clear cell renal cell carcinoma. Eur Radiol 30: 1274-1284, 2020.

59. Jeon MJ, Kim WG, Chung KW, Baek JH, Kim WB and Shong YK Active surveillance of papillary thyroid microcarcinoma: Where do we stand? Eur Thyroid J 8: 298-306, 2019.

60. Donovan JL: Presenting treatment options to men with clinically localized prostate cancer: The acceptability of active surveillance/monitoring. J Natl Cancer Inst Monogr 2012: 191-196, 2012.

61. Kim TY and Shong YK: Active surveillance of papillary thyroid microcarcinoma: A Mini-review from Korea. Endocrinol Metab (Seoul) 32: 399-406, 2017.

62. Ya M: Interpretation of the management guidelines for patients with thyroid nodules and differentiated thyroid cancer (2012 Chinese edition). Lin Chung Er Bi Yan Hou Tou Jing Wai Ke Za Zhi 27: 917-920, 2013 (In Chinese).

This work is licensed under a Creative Commons Attribution-NonCommercial-NoDerivatives 4.0 International (CC BY-NC-ND 4.0) License. 